\title{
Nouvelle image de la mère dans La Mayonnaise de l'écrivaine Jeon Hye-sung
}

\author{
Min Sook WANG-LE
}

\section{(2) OpenEdition}

Journals

Édition électronique

URL : http://journals.openedition.org/transtexts/499

DOI : 10.4000/transtexts.499

ISSN : 2105-2549

Éditeur

Gregory B. Lee

Référence électronique

Min Sook WANG-LE, "Nouvelle image de la mère dans La Mayonnaise de l'écrivaine Jeon Hye-sung », Transtext(e)s Transcultures 跨文本跨文化 [En ligne], 8 | 2013, mis en ligne le 02 décembre 2013, consulté le 19 avril 2019. URL : http://journals.openedition.org/transtexts/499 ; DOI : 10.4000/ transtexts.499 


\title{
Transtext(e)s Transcultures 跨文本跨文 1
}

Journal of Global Cultural Studies

$8 \mid 2013$ :

Genre et filiation : pratiques et représentations

Pères, mères, nourrices

\section{Nouvelle image de la mère dans La Mayonnaise de l'écrivaine Jeon Hye-sung}

\author{
MiN Sook WANG-LE
}

\section{Résumés}

Français English

Les écrivaines coréennes participent aujourd'hui à la transformation du monde littéraire et contribuent à travers leur écriture au changement de leur statut et de celui des autres femmes. Elles mettent en avant la complexité de l'image de la mère dans une nouvelle perspective. Tantôt rejetée, tantôt valorisée, la maternité devient chez certaines d'entre elles une matière privilégiée dans leur quête d'identité féminine. Cette étude privilégie le cas de l'écrivaine Jeon Hey-sung (1960-), auteur de La Mayonnaise, afin de comprendre comment elle envisage les rapports qu'entretiennent la mère et la fille. Le roman démystifie l'image de la mère sacrificielle et/ou asexuelle. La mère n'est pas ici dans une position de forme-sujet, vouée à la seule fonction de la maternité, mais celle d'un sujet indépendant qui assume son autonomie. L'écrivaine, par la voix de la narratrice, met plus en avant la féminité d'une femme que sa maternité.

Korean women writers today take part in the transformation of literary world and contribute through their works to the change of their own status and of other women's status. They highlight the complexity of the mother's image in a new perspective. Sometimes rejected, sometimes valued, motherhood becomes in some of them a preferred subject in the quest for their identity. This study emphasizes the case of the women writer Jeon Hey-sung (1960-), author of the Mayonnaise, with the aim of understanding how she envisages the relationships between mother and daughter. The novel demystifies the image of the sacrificial and/or asexual mother. The mother is not here in a position of form-subject, limited to the sole function of motherhood, but of an independent subject assuming her autonomy. By the voice of the narrator, the writer puts foremost the femininity of a woman before her motherhood. 


\section{Texte intégral}

Dans la tradition et jusqu'à aujourd'hui, les femmes coréennes exercent leurs principales activités autour et au sein de la famille. On sait que la dynastie de Joseon (1392-1910), la dernière dynastie de Corée, est fondée pour l'essentiel sur l'idéologie confucéenne qui distingue cinq types de relations sociales, celles qui relient le père au fils, le mari à la femme, le souverain au vassal, l'aîné aux cadets et celles entre les amis. En matière des femmes, elle privilégie une conception de "bonne mère et épouse vertueuse (hyeonmo yangcheo 賢母良妻) ». La stérilité d'une femme fut l'une des sept raisons de sa possible répudiation (chilgeo ji ak 七去之惡), les six autres étant : désobéissance aux beaux parents, jalousie, maladie incurable, bavardage, vol, débauche. Parmi les sept raisons qui causaient de nombreux drames, la stérilité, la maladie incurable et la jalousie étaient celles dont les femmes de Joseon subissaient le plus directement les conséquences.

2 Heo Nanseolheon 許蘭雪軒(1563-1589), célèbre poétesse du XVIe siècle, n'était pas considérée comme une mère accomplie car ses deux enfants (une fille et un fils) moururent tous les deux à leur tendre âge. Son talent poétique dépassait pourtant celui de ses frères et de son mari et fut connu même en Chine. ${ }^{1}$ Elle finit par se suicider à l'âge de 26 ans suite au conflit l'opposant à sa belle-mère et à son mari qui se remaria rapidement après sa mort pour avoir un fils héritier. Plus brillante que les hommes de son entourage mais incapable de donner à sa belle-famille un fils héritier, Heo Nanseolheon regretta d'être née femme à la dynastie de Joseon qui lui réservait un destin malheureux.

En se référant à l'idéologie confucéenne fondée sur le système patriarcal, la maternité fait naître en Corée les normes de l'éthique familiale imposant aux femmes d'enfanter un fils héritier, gage de la pérennité de la lignée et du culte des ancêtres. L'enfantement et l'éducation des enfants allaient de pair avec le sacrifice, le dévouement et la soumission. Autant une femme stérile pouvait se faire rejeter par sa belle-famille, autant une femme ayant un héritier avait la possibilité de profiter de la situation.

Cette conception traditionnelle marque aujourd'hui encore les mœurs de la société coréenne. Sin Saimdang 申師任堂 (1504-1551), mère du grand lettré Yul-gok (Yi I 李 珥,1537-1584), est associée depuis plusieurs siècles à cette image de mère exemplaire. Issue d'une famille noble, elle réunissait en elle toutes les qualités demandées par la haute société : poésie, broderie, peinture et calligraphie. Sa piété filiale fut légendaire à l'égard de ses propres parents et de ses beaux-parents. Elle se consacrait totalement à l'éducation de ses fils et de ses filles ( 7 enfants en tout). Elle fut l'exemple de toutes les femmes depuis le XVIe siècle jusqu'à la fin de la dynastie de Joseon. Aujourd'hui, elle est immortalisée grâce au billet de la plus importante coupure (50 ooo won) émise depuis 2009, son fils figurant depuis longtemps sur le billet de 5000 won.

Le thème de la maternité chez une grande partie d'écrivains hommes s'appuie largement sur l'image stéréotypée de Sin Saimdang. Cette façon de sacraliser la mère en tant qu'un être asexuel exclut paradoxalement l'expérience réelle de la maternité de la femme. En général, l'image de la maternité dans les romans coréens n'est pas libre de ces limites. Mais l'approche de certaines femmes écrivains contemporaines est tout autre. Elles cherchent à nuancer le thème de la maternité qui est souvent lié à leurs propres expériences. En phase avec l'évolution de la société coréenne, les femmes écrivains s'efforcent de se démarquer de l'image figée et fixe de la "bonne mère et épouse vertueuse ». Il ne s'agit pas, pour la plupart d'entre elles, de détruire totalement cette conception traditionnelle, mais de libérer le rôle de la mère d'un espace confiné, réduit à l'intérieur de l'unité familiale. Elles mettent en avant la complexité de l'image de la mère dans une nouvelle perspective. Tantôt rejetée, tantôt valorisée, la maternité devient chez 
certaines d'entre elles une matière privilégiée dans leur quête d'identité féminine. C'est cette nouvelle perspective, chère à Oh Jung-hee (1947- ), à Gong Seon-ok (1963-) et à Jeon Hye-sung (1960- ), qui attire notre attention. Leurs romans se différencient de ceux situés à mi-chemin entre l'amour et la compassion pour la mère, identifiée au pays natal, de ceux centrés sur l'évocation de la douceur maternelle mêlée de l'endurance et du dévouement et enfin de ceux attachés à l'idéalisation de la maternité. ${ }^{2}$ On voit chez $\mathrm{Oh}$ Jung-hee, écrivaine majeure qui excelle dans la nouvelle dès la fin des années 1960, la volonté d'assurer l'identité existentielle du « moi » par le rejet de l'image de la mère fondée sur le système patriarcal. Elle fut l'une des premières romancières à y introduire ce thème. Globalement, elle était traditionnaliste. Mais elle commençait à mettre en question l'image de " bonne mère et épouse vertueuse », promue par l'idéologie confucéenne sur laquelle s'appuyait la dynastie de Joseon. Oh Jung-hee représente un modèle de l'écriture féminine en Corée pour ses consœurs dont Jeon Hye-sung et Gong Seon-ok, actives depuis les années 1990 et qui traitent d'une façon plus complexe du sujet sur les femmes dans le cadre formel et social. ${ }^{3}$

Cette étude privilégie le cas de l'écrivaine Jeon Hey-sung, auteur de La Mayonnaise, afin de comprendre comment elle envisage les rapports qu'entretiennent la mère et la fille. Le roman démystifie l'image de la mère sacrificielle et/ou asexuelle. La mère n'est pas ici vouée à la seule fonction de la maternité, mais dans la position d'un sujet indépendant qui assume son autonomie.

\section{Jeon Hye-sung et La Mayonnaise (마요 네즈)}

Née à Pusan en 1960, Jeon Hye-sung est diplômée d'un master en philosophie de la célèbre Université des Femmes Ewha. Après avoir été journaliste pour les magazines cinématographiques, elle a commencé sa carrière d'écrivain par la dramaturgie en écrivant des pièces de théâtre. La Mayonnaise est son premier roman. Dès sa publication en 1997, l'œuvre a remporté le prix littéraire « Munhak dongne » 문학동네 (Village de la littérature) des jeunes écrivains. Le roman est caractérisé par des détails, des décors et une description fine de la psychologie des personnages, ce qui le dote d'un effet de théâtralité. En 1999, l'écrivaine a réalisé elle-même son adaptation en scénario. Le film qui porte toujours le même nom, réalisé par Yun In-ho, a été couronné en $2000 \mathrm{du}$ Golden Crow Pheasant Award au Festival international du film à Kerala, en Inde. La relation entre la mère et la fille est projetée chez Jeon Hye-sung au premier plan dans l'organisation narratrice, ce qui n'est pas étranger dans le récit à caractère autobiographique. ${ }^{4}$ Mais la question de savoir s'il s'agit d'un récit autobiographique est secondaire par rapport à la riche leçon philosophique que revêt le roman.

L'histoire de La Mayonnaise commence par l'arrivée de la mère, âgée de 63 ans, chez la fille-narratrice A-jeong, âgée de 36 ans, pour y vivre ensemble. La mère, dont la beauté n'avait autrefois rien à envier à celle d'Elisabeth Taylor, actrice hollywoodienne, est à présent épuisée psychiquement et physiquement par la violence et la faillite économique du père, décédé peu de temps auparavant. Dépendante à l'insuline qu'elle s'injecte et à d'autres médicaments, son état de santé provoque de la compassion chez la fille.

Mais la narration s'attarde sur la relation conflictuelle entre mère et fille. La présence de la mère trouble le rythme de la vie familiale et professionnelle de la fille, mère ellemême de deux enfants et qui a un petit travail de nègre rédigeant une biographie pour une femme devenue célèbre courtière d'assurances en élevant toute seule ses enfants. Cette mère de 53 ans a réussi à envoyer ses quatre enfants, moteur de son courage de vie 
après le décès soudain de son mari, pour étudier à l'étranger : trois aux États-Unis et un en Chine. C'est une mère sacrificielle qui s'inscrit dans la droite ligne de Sin Saimdang et qui contraste totalement avec sa propre mère qui occupe une place pesante chez elle. Ajeong doit se confronter quotidiennement à deux images de mère à l'intérieur de son appartement : dans son bureau où elle écrit ou retranscrit l'histoire de la courtière d'assurances après l'avoir à maintes fois interviewée et dans une chambre occupée sa propre mère, qui ne s'intéresse qu'à ses propres affaires.

Elle trouve sa mère égoïste. Celle-ci essaie de satisfaire seulement son désir plutôt que d'aider sa fille et de s'occuper de ses deux petits-enfants, âgés respectivement de 4 ans (Hae-mi) et de 6 ans (Hae-gi). Au contraire, elle pense que sa fille ne prend pas assez soin d'elle et que ses deux petits-enfants sont embêtants. Devant sa fille fatiguée par la tâche de ménage et par le travail de rédaction, la mère s'apitoie sans cesse sur son propre sort de ne pas pouvoir avoir un mari tendre et un fils héritier chez qui elle aurait pu tranquillement être servie par sa belle-fille. Ayant seulement trois filles, elle n'a pas selon elle une vie confortable que ses amies ont grâce à leurs fils.

A-jeong supporte mal sa mère râleuse et qui ne cherche que du confort. Angoissée par sa présence, elle cherche néanmoins à échapper au poids du présent dans ses souvenirs d'enfance, de jeunesse passée dans la maison maternelle. Mais ils ne sont pas plus euphoriques : sa mère qui préparait devant le miroir son maquillage pour sortir tous les soirs, son père pharmacien non certifié qui tapait violemment la mère infidèle et ses trois filles (elle et les deux petites sœurs), victimes de ce conflit parental. La maison familiale n'était qu'un espace destructeur :
Presque tous les jours, ma mère ne rentrait que très tardivement [à la maison] après avoir passé son temps avec des inconnus dans des endroits imprévisibles. L'attente de son retour pour lequel je comptais seconde par seconde me demandait une énergie débordante, destructrice de mon âme. Lorsqu'elle ne revenait pas après 11 heures du soir, j'étais saisie d'une frayeur à l'idée qu'elle ne reviendrait peut-être plus jamais. Le regard aux flammes bleues de mon père flamboyait comme la mèche d'une bougie. Les bras croisés, les sourcils noirs froncés, l'air menaçant, il me fit penser à un garde coléreux.

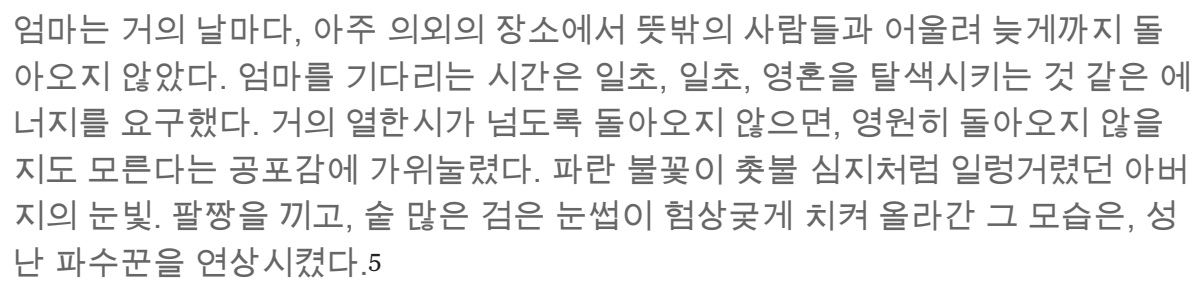

La narratrice se sentait très tôt différente de sa mère qui aimait profiter de la vie au lieu de rester à la maison pour son mari et ses enfants. À l'opposé de sa chambre décorée de livres, celle de sa mère est remplie d'objets de femme :
De même que ma mère se sentait mal à l'aise dans ma chambre où j’étudiais, j'étais déprimée par sa somptueuse couverture, le bout de pinceau rose coagulé d'une manucure sirupeuse et sensuelle, son pinceau de maquillage doux et facile à manier à force d'utilisation, sa fourrure de peau de renard, ses bagues ornées de saphir et de perles, et enfin tous ces innombrables objets étranges, vaniteux et qui se trouvaient [pêle-mêle] dans sa chambre à coucher.
엄마가 내 공부방에서 주눅이 들었듯이, 나는 침대방의 화려한 이불이며, 매니큐 어의 요염한 끈적거림이 엉겨붙은 핑크빛 붓끝, 탐스럽고 부드럽게 길이 잘 든 화 장솔이며, 여우목도리, 사파이어반지와 진주목걸이, 그 밖에도 헤일 수 없이 잡다 한, 엄마의 야릇하고 교만한 물건들 앞에서 풀이 죽었다. 6

Les deux types d'espaces, la chambre d'A-jeong et celle de sa mère, représentent ici 
deux modes de vie et deux états d'âme complètement différents. Ils sont incompatibles dans la mesure où les deux occupantes du lieu sont enfermées l'une comme l'autre dans leur propre univers sans une compréhension mutuelle. Elles n'ont pas de valeurs partagées à part leur relation de filiation. C'est cet antagonisme entre la mère et la fille, déjà installé dans la maison maternelle de la narratrice, qui constitue le cœur du roman.

\section{L'incident de la mayonnaise et le conflit entre mère et fille}

Toutes les mères ne sont pas aimantes, toutes les écrivaines ne célèbrent pas les péripéties aboutissant à une réconciliation entre mère et fille. Si l'on suit la chronologie du roman, la relation conflictuelle entre mère et fille arrive à son sommet dans ce que la narratrice nomme « l'incident de la mayonnaise ».

Lorsqu'A-jeong est allée chez ses parents, juste après son mariage, pour voir son père complètement anéanti à la suite d'une attaque d'apoplexie, sa mère se plaignait que son mari malade ne mourût pas assez vite devant la fille qui ne cessait de pleurer. Et une odeur aigre et fétide lui est parvenue de la tête de sa mère à côté de son père alité qui a déféqué dans son pantalon :

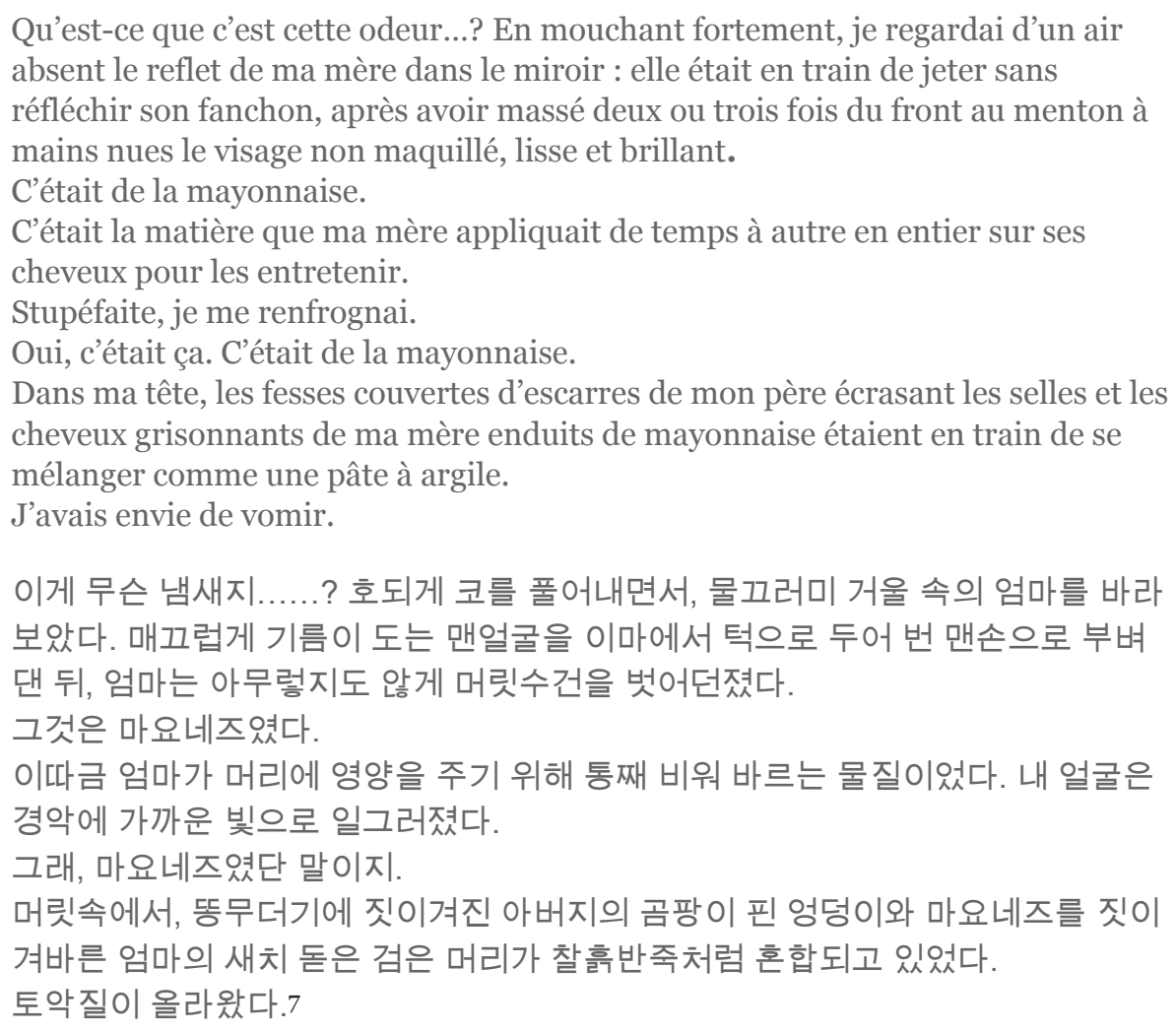

Ce passage qui évoque pour la première fois « l'incident de la mayonnaise » est très important dans le récit. Pour la narratrice, la mayonnaise, utilisée comme produit de beauté par sa mère, et les selles de son père sont sur le même plan : refusant de soigner son mari mourant et le confiant à une soignante sous prétexte de sa propre faiblesse, la mère n'oublie pas ses propres soins de beauté. La mayonnaise fonctionne ici comme une métaphore qui symbolise le désir égoïste et déformé de la mère. Depuis que la narratrice a assisté à cette scène de l'usage de la mayonnaise comme une matière esthétique et non comme de la nourriture, ce que feraient la plupart des mères de famille, elle éprouve un 
profond mépris envers sa mère. Elle a du mal à s'imaginer que pour sa mère, entretenir sa chevelure est une chose plus importante que de nourrir ses enfants et de soigner son mari mourant.

Cette histoire de la mayonnaise a un impact direct et durable sur la relation entre la mère et la fille. Cette dernière constate que sa mère, logée chez elle, qui a même des difficultés à se tenir en équilibre, continue malgré son âge à s'enduire la tête de mayonnaise pour soigner ses cheveux. La fille retrouve un soir, après une rencontre avec la courtière d'assurances, au retour à la maison sa mère qui s'évanouit dans la salle de bain, la tête couverte de mayonnaise, sans s'occuper de ses deux enfants :
C'était de la mayonnaise. La porte crémée du réfrigérateur, placé en face de la porte de la salle de bain, était entièrement ouverte. Un peu plus loin, le tube de mayonnaise était laissé par terre, écrasé à plat et privé du couvercle de couleur jaune. Je ne pouvais pas deviner quand elle avait perdu l'équilibre, à quel moment elle avait fini par tomber. Mais je pouvais imaginer dans ma tête, comme si je voyais un film, les étapes : elle se fit un shampooing, s'enduit les cheveux de mayonnaise et tenta de se rincer les cheveux avec la pomme de douche.
그것은 마요네즈였다. 욕탕문 비껴 맞은편, 냉장고의 크림색 문짝도 벌렁 열려 있 었다. 그 앞, 마요네즈병이 노란 뚜껑이 날아간 채 납쭉 찌그러져 팽개쳐져 있다. 언제 균형을 잃고 자빠지고 말았는지까진 헤아릴 순 없었다. 하지만 샴푸로 머리 를 감은 후, 마요네즈를 바르고, 다시 샤워기로 그것을 헹궈내려고 했을 과정들만 은 영화를 보듯 환히 그려졌다. 8

Si la mère a maintenant changé de cadre de vie - elle se trouve chez sa fille -, son habitude reste inchangée et ce malgré son âge. La mayonnaise comme le fil conducteur du récit revient ici comme un refrain. La théâtralité du roman se traduit ainsi par plusieurs actes rythmés à chaque fois par ce thème central de la mayonnaise, d'où le titre du roman qui se justifie sur plusieurs plans. En même temps, le thème de la mayonnaise ne cesse d'être approfondi au niveau psychologique de la part de la narratrice. L'incompréhension ou l'incommunicabilité se transforment au fur et à mesure en haine jusqu'ici refoulée ou masquée :

Je n'avais pas envie de lui faire sa toilette. Mais je ne voulais pas non plus sentir cette odeur, ni ouvrir les fenêtres pour aérer l'appartement. Je n'éprouvais même pas de pitié pour ses cheveux tressés derrière les oreilles en sueur. Je fus envahie par un sentiment d'esclave à cause de ce lien de sang, un lien malheureux qui semblait être mon karma.

«Que faire? Que faire de cet ennemi? Il va à coup sûr me détruire avant qu'il ne meure... »

Quand, à qui, qui avait-elle chargé de cette malédiction?

Au moment où je sentis l'odeur de la mayonnaise, j'avais l'impression que le sang de tout mon corps refluait. Elle me fit penser au malheur de mon père en débâcles.

Voyant ma mère avec du cold-cream sur son visage, plus luisant que l'eau excrémenteuse [de mon père], j'eus une sensation de démangeaisons au dos comme si une scolopendre s'y était tortillée. Pendant plusieurs mois, je n'arrivais pas à mettre la mayonnaise dans la nourriture. La mayonnaise ne me semblait plus une simple sauce de salade mais un symbole tragique qui liait ma mère à mon père.

Je finis par jeter dans le sac poubelle le tube de mayonnaise dont le bouchon avait été ouvert. Pendant un long moment, je tremblais de colère, me tenant debout, les paumes posées aux bords de la table à manger. J'avais envie au moins de crier. Mais ça n'avait aucun sens tant que ma mère restait à côté de moi. Je sanglotais tristement. 


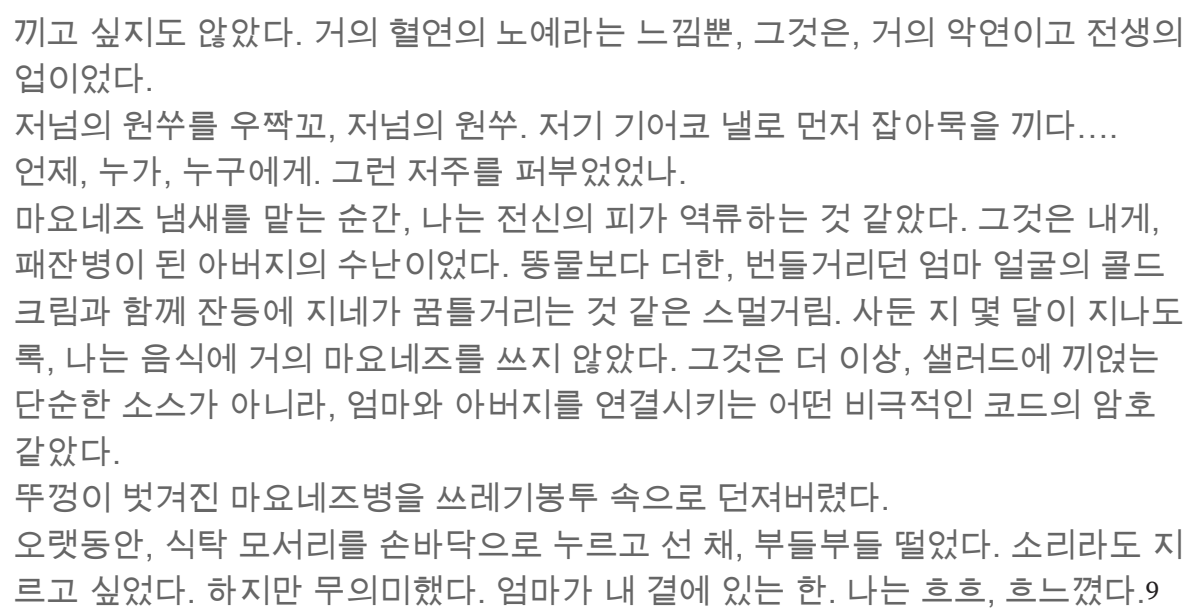

Cet incident de sa mère, lié de nouveau à la mayonnaise, lui rappelle un souvenir ancien : la souffrance de son père. Même si l'amour que sa mère lui a témoigné est gravé dans sa mémoire, elle éprouve de la honte envers son irresponsabilité car elle ne s'intéresse pas à ses petits-enfants, dont elle ne s'occupe pas, pas plus que de la maison, ne s'attachant qu'à son corps, déjà vieilli et maladif. Jeter le tube de mayonnaise qui symbolise l'image de sa mère signifie l'envie d'A-jeong de la supprimer, de « détruire cet ennemi » comme le dit la citation. Ce passage traduit un point culminant de la tension entre la mère et la fille. Mais ce projet d'élimination de sa mère est-il réalisable ? Non. Les choses paraissent plus compliquées et complexes. En effet, le texte l'annonce implicitement en comparant les relations entre mère et fille à un karma, inscrit dans le destin de la narratrice. Celles-ci ressemblent d'ailleurs à la mayonnaise aigre et poisseuse qui ne peut se délier facilement. Une filiation peut être reniée subjectivement. Il n'empêche que ce soit un lien de sang qui revêt une objectivité. Dans le passage suivant, la fille déclare en sanglots la séparation avec elle :

«Maman, je ne peux plus te supporter... »

Je rangeai de ma main les cheveux dispersés.

Ma mère, les yeux grandement ouverts, était abandonnée à côté de moi.

« Eh ! Aide-moi à me relever s'il te plaît. »

«Maman, je, je ne pourrais vraiment pas vivre avec toi... »

Malgré moi, mon âme dans sa profondeur pleurnichait.

« Eh ! ... J'ai quelque chose à te dire. Relève-moi, s'il te plaît... »

« Maman, serait-il possible que tu rentres chez toi? »

Mon fantôme dévoilait ainsi ma vraie intention.

Lorsque je voulais marcher vers ma mère en sanglotant, j'étais prise d'un vertige côté occiput. J'avais mal partout comme si j'avais reçu un coup de marteau en fer et j'étais dans une panique la plus totale. Je passai autour de ma mère et entrai en titubant dans ma chambre. Je ne pouvais pas savoir comment j'avais réussi à déplier la couverture. Je remerciais même Dieu de me mettre la couverture qui me permettait de me coucher. Je fermai les yeux. Et je n'avais plus envie de me réveiller.

J'étais parfaitement tombée dans le piège tendu par le démon.

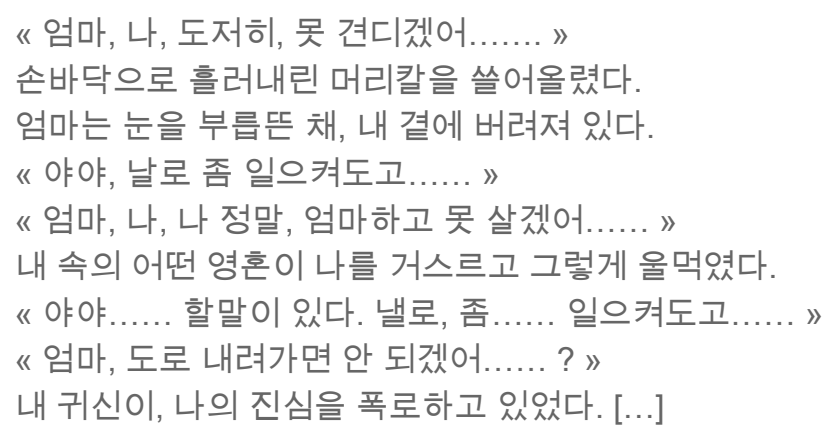




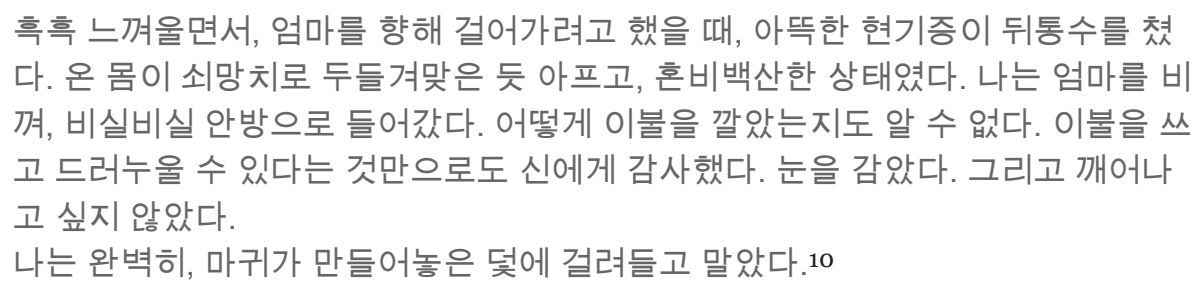
a littéralement laissé tomber sa mère, toujours couchée sur le sol de la salle de bain. Le dialogue suivant continue à illustrer l'incommunicabilité de ces deux femmes :

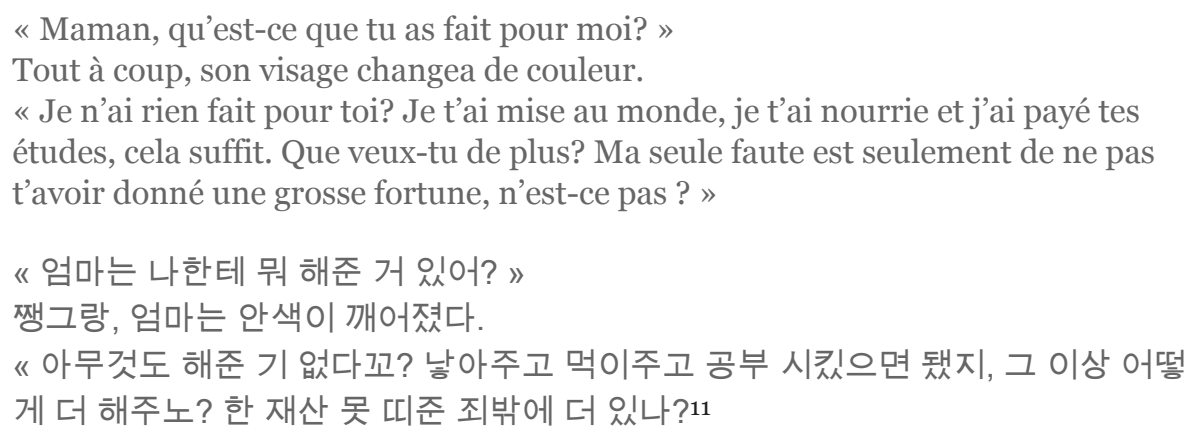

Dans le développement du roman, on peut s'attendre à un blocage, à ce que cette situation aboutisse à une impasse, ou à voir la mère rentrer chez elle. Mais le récit introduit un tournant en faisant appel à un autre récit, à une intertextualité grâce à une évocation de l'histoire vécue par la mère. Jusque-là, on voit qu'il existe un écart des points de vue : celui de la mère est différent de celui de la fille. La fille apprécie une mère traditionnelle alors que la mère elle-même aspire à une liberté, à sa propre autonomie. Pour la comprendre, il faut d'abord respecter sa position de femme, avec sa féminité, qui passe par-dessus le devoir familial.

\section{Issue du conflit : vers une réconciliation entre mère et fille}

C'est le moment où A-jeong demande à sa mère de partir que celle-ci livre son propre récit. En effet, la grand-mère maternelle était une personne qui a cherché seulement le bonheur personnel dans ses rôles respectifs de troisième épouse d'un homme riche mais âgé, de veuve infidèle et encore d'épouse d'un veuf. Elle était un contre exemple par excellence d'une mère sacrificielle. Profondément blessée par l'absence répétée de sa mère, la mère d'A-jeong lui faisait toujours reproche de sa frivolité et son irresponsabilité, au lieu d'essayer de comprendre la grand-mère qui ne pouvait vivre que de cette façon. Leur relation était extrêmement tendue :

\footnotetext{
J'avais envie de mourir de honte de ta grand-mère. J'avais tellement honte que je souhaitais fort qu'elle mourût. À cette époque, il était quasiment impossible d'imaginer que les femmes se remariassent. Maintenant, à la réflexion, j’ai tant de remords. J'aurais dû lui trouver quelqu'un pour elle, mais je ne prononçais que des injures derrière ta grand-mère qui était partie en me disant qu'elle ne voulait plus me voir.

나는 할머니가 똑 챙피해서 죽고 싶었다. 우찌 수치스럽고 챙피스럽던지, 할머이 가 마, 팍 죽어붔으면 좋겠다꼬 생각했다. 그때만 해도, 여자가 호적을 파서, 딴 집 에 간다는 거는 여간해서는 상상을 못 했다. 지금 생각하면, 그래 후회스러블 수가 없다. 내가 나서서 할머이 결혼을 시키드맀어도 부족했을 낀데....... 다시는 얼굴
} 


\section{도 보기 싫다고, 떠나는 할머이 뒤꼭대기에 대고 패악만 안 부맀나.12} malheureuse en élevant elle-même une fille en tant que mère et femme. Cette compréhension n'est possible pour elle qu'après avoir successivement joué trois rôles au gré des âges de la vie : jeune fille, mère et grand-mère :

Ma mère ne cessait de défaire le rouleau de papier hygiénique.

« Mais, c'est la vie. À quoi ça sert de regretter comme ça maintenant ? Quand je mourrai, j'espère que je rencontrerai une fois ta grand-mère. Alors, je lui demanderai pardon pour tout ce que j'ai fait contre elle. [...]

« Oui, c'est dur pour toi à cause de moi ? »

J'enfonçais sans arrêt mes doigts dans les cheveux. J'ai voulu la consoler, mais aucun mot n'est sorti de ma bouche. C'est ma mauvaise habitude. Je n'ai même pas essayé une seule fois d'incorporer sa vie dans la mienne.

« Je ne te cause que des ennuis. Je n'ai même pas soutenu jusqu'au bout tes études que tu voulais continuer. Lorsque tu t'es mariée, je ne t’ai pas donné grand-chose. Je ne t'ai pas donné non plus grand-chose quand tu avais accouché. Tout le temps, tu t'es donné de la peine pour moi et ton père. Une mère pareille comme moi n'existerait pas dans ce monde... »

Je m'étouffe toute seule. Je ne mérite pas de recevoir ses excuses unilatérales.

« Je dois te quitter... »

Eh ! Maman...

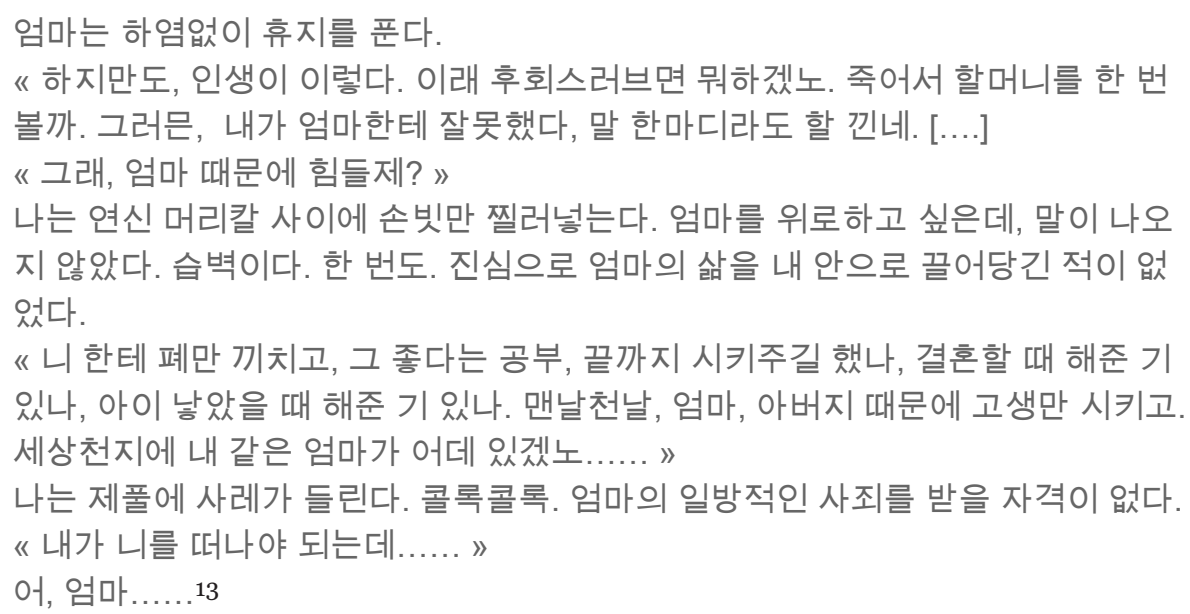

Le rappel de l'histoire vécue de la mère prend de plus en plus de poids dans la narration. A-jeong n'est pas habituée aux mea-culpa de sa mère. La révélation mêlée de remords de la mère, qui n'accepte pas d'abandonner entièrement sa propre vie au profit des enfants et des petits-enfants, calme l'émotion de la fille-narratrice, qui était sur le point de congédier sa mère. Elle parvient à comprendre la vie circulaire des femmes dans sa continuité spirale de " grand-mère $\rightarrow$ mère $\rightarrow$ fille », en se mettant à la place de sa grand-mère et à celle de sa mère :

Ma mère qui se trouvait devant moi ne me paraissait plus être la même. En elle qui était issue du corps de sa mère [ma grand-mère] transparaissait le visage traduisant mon futur vieillissement solitaire. Elle évoquait une élégie solitaire que je chanterai pour moi-même, lorsque je serai âgée, devant ma fille Hae-mi : la vie est une répétition sans fin.

내 앞의 엄마가 엄마로 보이지 않았다. 엄마의 껍질을 찢고 나온 엄마는, 먼 훗날 홀로 노쇠해갈 내 얼굴처럼 보였다. 혹은 아주 늙어버린 내가, 해미를 앉혀놓고 부 르는 외로운 만가 같았다. 삶은 끝없는 되갚음. 14

L'état présent de la mère évoque le passé (grand-mère) et le futur (moi-même et ma fille même). Les trois dimensions du temps sont ici fusionnées en la personne de la mère 
qui représente une certaine généralité de la vie d'une femme. On passe ici d'un registre particulier à un registre plus général. Les aveux imprégnés de remords de la mère, qui supportait mal la grand-mère qui n'avait pas abandonné sa propre vie malgré une grande affection pour elle, provoquent chez la fille-narratrice, un éveil. Elle comprend enfin qu'une femme peut avoir des rôles différents selon leurs positions de grand-mère, de mère et de fille. La maternité et la féminité ne sont pas toujours dans une relation harmonieuse. En tant que grand-mère ou mère, elle est souvent réduite à une fonction nourricière ou éducatrice ; mais en tant que femme, elle peut aussi aspirer à son propre bonheur. Dans le rôle fonctionnel de la grand-mère ou de la mère, elle subit, fait ce qui correspond à l'attente de la société ; mais en tant que sujet en quête de son identité, de son bonheur, elle peut aussi agir librement. Mais il y a un prix à payer lorsque les conditions socio-économiques ne sont pas réunies. La mère d'A-jeong est obligée d'aller vivre avec sa fille sans moyens matériels. C'est cette distance entre l'hétéronomie et l'autonomie qui rend la réalité complexe et le conflit entre mère et fille tendu.

Le roman n'a pas donné une réponse à la façon de résoudre ce type de relation conflictuelle entre mère et fille, mais suggère en dernier ressort une solidarité des femmes, une compréhension possible inter-générationnelle malgré une teinte de pessimisme :

\begin{abstract}
Mais je ne changerai pas vraiment. Les changements ayant lieu ne se sont jamais opérés dans le sens souhaité. Ma mère ne changera pas tellement non plus. Elle ne sait même pas quel changement il nous faudrait. Dès le début, la vie était pour elle une jouissance mais pas une méditation. C'est pour cela que je ne devrais pas être désespérée vis-à-vis des choses inchangeables. Il ne me reste plus de force pour arrêter une roue de char déjà engagée sur une pente. Si, dès le départ, il n'y avait aucune attente réciproque dans nos liens, c'aurait pu être mieux.

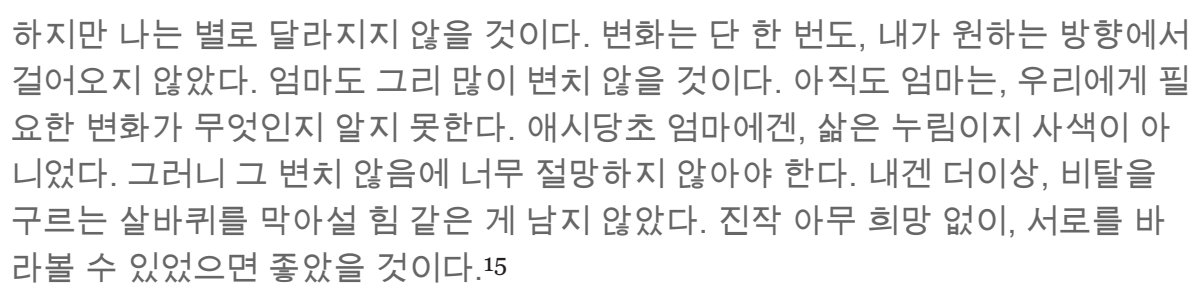

Face à sa grand-mère et à sa mère qui se livrent à la quête de leur propre bonheur, Ajeong commence à les comprendre mais ne s'engage pas in fine à suivre leur mode de vie. La réconciliation entre mère et fille ne se joue pas par conviction (chacune continue à garder son habitus) mais par une acceptation du destin familial (de la grand-mère à la mère), de la « roue » karmique par la fille. Elle est dans ce sens fataliste.

\title{
Conclusion
}

Dans La Mayonnaise, Jeon Hey-sung dépeint avec finesse les rapports conflictuels entre A-jeong et sa mère, qui est loin d'être fidèle à l'image de la mère traditionnelle : sacrificielle et/ou asexuelle. Celle-ci est plus préoccupée par son rôle de femme que par celui de mère. Autrement dit, la féminité est plus importante pour elle que la maternité, et le fait de devenir mère ne signifie pas la perte d'autonomie dans sa vie de femme. Mais une autonomie nécessite des conditions socio-économiques, ce qui n'est pas le cas de la mère d'A-jeong et peut-être aussi de la plupart des femmes de sa génération. C'est lorsqu'elle vient habiter chez sa fille après avoir perdu son mari que le conflit entre mère et fille atteint son plus haut degré. Le roman se termine par un dénouement heureux : la réconciliation entre mère et fille. Mais cela demande à la fille un renoncement à des attentes affectives vis-à-vis de sa mère, une acceptation du statu quo voire même une 
adhésion à un certain déterminisme relatif à la filiation.

L'enjeu du roman est sans doute ailleurs. En mettant en scène une telle mère fidèle à son propre désir de femme, mal vue du point de vue patriarcal traditionnel et difficilement acceptée par sa propre fille, l'écrivaine lui prête une voix pleine, une voix que l'on n'entend pas ailleurs, du moins pas habituellement. Avec la démystification de l'image de la mère fabriquée de toutes pièces par la société coréenne traditionnelle, ce roman de Jeon Hye-sung s'approche d'une interprétation réelle de la vie d'une mère. Il rend compte des facettes multiples qu'elle peut avoir. La Mayonnaise ne cesse de confronter le point de vue de la mère et celui de sa fille. L'écart entre les deux est grand et ne se dissipe pas à la fin du récit. L'écrivaine n'a pas de parti pris ni pour l'une ni pour l'autre. Elle laisse entendre qu'on ne doit pas se limiter à un seul regard car être mère dans une société moderne est une réalité complexe et ambiguë. L'identifier à une image figée, qu'elle soit sacrificielle ou égoïste, n'est pas une démarche à préconiser.

\section{Notes}

1 L'un de ses frères est Heo Gyun (1569-1618), lettré et auteur du Hong Gil-dong jeong 홍길동 전(Histoire de Hong Gil-dong), premier roman écrit en coréen, alors que le premier roman en chinois, le Geumo sinhwa 金鰲新話 (Nouveaux contes du Mont de la Tortue d'or) est dû à Kim Si-seup (1435-1493).

2 Voir pour plus de détails : Groupe de recherche de la littérature féminine à l'Université de Sogang, Hanguk munhak-kwa moseong seong (La Maternité dans la littérature coréenne), Séoul, Taehak-sa, 1998.

3 Sim Jin-gyeong, Yeoseong, munha-geul garojireuda, (Les Femmes qui traversent la littérature), Séoul, Munhak-kwa jiseong-sa, 2005, p. 183.

4 «La présence de la mère prend inévitablement pour les femmes un autre sens que pour les hommes, puisque leur mère est leur exacte matrice, leur préfiguration », Béatrice Didier, L'écriture-femme, Paris, PUF, 1991, p. 5.

5 Jeong Hye-sung, Le Mayonejeu (La Mayonnaise), Séoul, Munhak Dongne, 1997, p. 101-102.

6 Jeong Hye-sung, op.cit., p. 97-98.

7 Jeong Hye-sung, op.cit., p. 158.

8 Jeong Hye-sung, op.cit., p. 179.

9 Jeong Hye-sung, op.cit., p. 180-181.

10 Jeong Hye-sung, op.cit., p. 181-182.

11 Jeong Hye-sung, op.cit., p. 186-187.

12 Jeong Hye-sung, op.cit., p. 195.

13 Jeong Hye-sung, op.cit., p. 197.

14 Jeong Hye-sung, op.cit., p. 191.

15 Jeong Hye-sung, op.cit., p. 200.

\section{Pour citer cet article}

Référence électronique

Min Sook WANG-LE, « Nouvelle image de la mère dans La Mayonnaise de l'écrivaine Jeon Hye-sung », Transtext(e)s Transcultures 跨文本跨文化 [En ligne], 8 | 2013, mis en ligne le 02 décembre 2013, consulté le 16 janvier 2014. URL : http://transtexts.revues.org/499

\section{Auteur}

Min Sook WANG-LE 
Auteur d'une thèse sur Colette (Récit et saison chez Colette, L'Harmattan, 2001) et d'une autre sur l'écrivain coréen Kim Dong-ri (1913-1995), Min Sook WANG-LE est maître de conférences en langue et culture coréennes à l'Université Jean Moulin Lyon 3. Sa recherche actuelle porte sur la littérature coréenne moderne et contemporaine.

\section{Droits d'auteur}

(C) Tous droits réservés 